FORMATION Formation emploi

Revue française de sciences sociales

114 | Avril-juin 2011

Pêle-mêle

\title{
L'indice de concentration : une clé pour analyser l'insertion professionnelle et évaluer les formations
}

The concentration index: a key to analysing employability and evaluating training

Der Konzentrationsindex: Schlüssel für die Analyse der beruflichen

Eingliederung und die Evaluierung der Ausbildungen

El índice de concentración: une clave para analizar la inserción profesional y evaluar las formaciones

\section{Catherine Béduwé et Jean Vincens}

\section{OpenEdition}

Journals

Édition électronique

URL : http://journals.openedition.org/formationemploi/3320

DOI : 10.4000/formationemploi.3320

ISSN : 2107-0946

Éditeur

La Documentation française

Édition imprimée

Date de publication : 31 juillet 2011

Pagination : $5-24$

ISSN : 0759-6340

Référence électronique

Catherine Béduwé et Jean Vincens, «L'indice de concentration : une clé pour analyser l'insertion professionnelle et évaluer les formations », Formation emploi [En ligne], 114 | Avril-juin 2011, mis en ligne le 04 octobre 2011, consulté le 30 octobre 2020. URL : http://journals.openedition.org/ formationemploi/3320 ; DOI : https://doi.org/10.4000/formationemploi.3320 


\title{
Accès à l'emploi \\ et insertion
}

\section{L'Indice de concentration: une clé pour analyser l'insertion professionnelle et évaluer les formations}

\author{
Catherine Béduwé et Jean Vincens*
}

L'indice de concentration des emplois occupés caractérise l'insertion professionnelle des sortants d'une formation. Plus l'indice est élevé, plus les jeunes ont pu faire valoir leur avantage compétitif et accéder aux meilleurs emplois parmi ceux qui leur étaient accessibles.

L'évaluation externe des formations, c'est-à-dire à travers l'insertion professionnelle des diplômés, est inscrite dans la loi LRU de $2007^{1}$. Évaluer pour mieux former et mieux informer les étudiants devient une mission attribuée aux universités. L'objectif de cette mission semble clair et de bon sens. Cependant, la complexité des relations formation-emploi rend cette démarche d'évaluation relativement technique et le sujet polémique.

\footnotetext{
${ }^{1}$ La loi du 10 aout 2007 sur les « Libertés et les Responsabilités des Universités », dite loi LRU, prévoit (article 20) "que l'article L612-1 du code de l'éducation est complété par un alinéa : Les établissements dispensant des formations sanctionnées par un diplôme d'études supérieures rendent publiques des statistiques comportant des indications de réussite aux examens et aux diplômes, de poursuite d'études et d'insertion professionnelle des étudiants ».
}

* Jean Vincens est professeur émérite d'économie à I'université Toulouse 1 Capitole. II a récemment publié : "Évolution de l'enseignement supérieur : persistances et paradoxes », Cahier du Lirhe n 14, 260 p.

Catherine Béduwé est ingénieur de recherche au CRM (Centre de Recherches en Management) de l'université de Toulouse 1 Capitole. Dernières publications: "Mismatch of vocational graduates what penalty on French labour market? », with J.F. Giret, Journal of Vocational behavior (2010), doi/10.1016:J;JVB;2010.09.003 et " Le sentiment d'insécurité : quelle cohérence entre les données objectives et la subjectivité des acteurs ? » en collaboration $\operatorname{avec} \mathrm{A}$. El Akremi, in B. Conter, P. Lemistre et B. Reynes, L'ancienneté professionnelle à l'épreuve de la flexicuritée, Presses de l'Université des Sciences Sociales de Toulouse. 
Par opposition à l'évaluation interne qui concerne le contenu du cursus de formation, les méthodes pédagogiques et les vérifications des acquis, l'évaluation externe porte sur les résultats obtenus par les formés sur le marché du travail. Ainsi la formation, en tant que dispositif institutionnalisé, peut être évaluée à l'aide des critères utilisés par les jeunes eux-mêmes pour apprécier leur réussite. Dans cette perspective, le temps d'accès à l'emploi des diplômés, le salaire obtenu, le niveau et la stabilité de leur position professionnelle, la satisfaction qu'ils en tirent sont des critères couramment utilisés pour évaluer une formation. Plus ces critères présentent des taux élevés, plus la formation aura atteint ses objectifs d'insertion professionnelle et mieux elle sera jugée. Ces informations sont censées permettre aux étudiants des générations suivantes de mieux s'orienter et aux universités d'améliorer, le cas échéant, leur offre de diplômes.

Cette démarche d'évaluation procède d'un postulat, à savoir que la formation joue un rôle important voire déterminant dans la réussite de l'insertion professionnelle des individus. Lorsque l'objectif d'insertion n'est pas atteint, c'est-à-dire lorsque les jeunes issus d'une formation connaissent chômage, précarité, déclassement, ... on suppose implicitement que la formation est très largement responsable de ces mauvaises performances. La publication de ces résultats doit inciter les étudiants à choisir leur formation en toute connaissance de cause, et les universités à opérer les changements nécessaires pour améliorer ces résultats. Or deux problèmes se posent :

Le premier est que les compétences d'un individu ne se limitent pas à sa formation initiale et que, par ailleurs, une série de variables personnelles et contextuelles influencent l'utilisation des compétences possédées. L'influence de la formation est donc toujours relative. Il convient d'en tenir compte et de comprendre en quoi les compétences acquises en formation permettent aux diplômés de s'insérer professionnellement, avant de porter le moindre jugement de valeur sur la formation.

Le second problème est que l'insertion professionnelle est multidimensionnelle et que les différents critères de réussite utilisés pour l'apprécier sont faiblement corrélés (Béduwé Espinasse 2009). Il n'existe donc pas d'indicateur qui résume la réussite de l'insertion professionnelle. En effet, les jeunes sont contraints de faire des choix en entrant sur le marché du travail et d'ordonner leurs préférences. Les sortants d'une formation peuvent par exemple éviter le chômage en acceptant des emplois en dessous de leurs espérances ou encore obtenir un meilleur salaire mais au prix d'une situation précaire. Dès lors, toute évaluation des formations selon un critère unique, privilégié par l'évaluateur, méconnaît le principe même de l'évaluation externe qui repose sur le constat de cette multidimensionnalité de l'insertion professionnelle. Le classement des formations ou encore des universités ${ }^{2}$, selon ce type d'évaluation, va donc dépendre de l'indicateur choisi et de la prise en compte plus ou moins soigneuse des effets individuels ou contextuels de l'insertion (voir par exemple Bourdon, Giret, Goudard, 2011). Ces questions sont d'importance puisqu'une partie des financements des universités est amenée à dépendre de cette évaluation des performances des formations et donc des établissements qui les dispensent.

Le choix des critères pour évaluer l'insertion professionnelle, que nous appelons critères de résultat, est donc une étape importante. Ils peuvent être classés en deux catégories : ceux qui traitent de l'accès des jeunes à l'emploi en général et ceux qui mesurent l'accès des jeunes à des emplois particuliers. Les premiers sont relativement consensuels parce qu'ils objectivent ce qu'on suppose être les choix de la plupart des jeunes : trouver rapidement du travail, trouver un emploi stable plutôt que précaire et/ou un emploi bien rémunéré. Les seconds s'inscrivent dans une problématique des relations entre formations et emplois, principalement développée par la recherche et la pratique à partir de la notion de correspondance. Celle-ci peut prendre deux formes :

La première est celle de la correspondance entre les niveaux de formation et les niveaux d'emploi pour mesurer la suréducation et le déclassement. Cette approche a donné lieu à une abondante littérature (Mc Guiness, 2006 ; Plassard et Thi Thanh Nhu Tra, 2009 ; Cedefop 2010 pour des revues de littérature récentes). La seconde porte sur la mise en relation des

\footnotetext{
${ }^{2}$ Voir le classement des universités publié en 2010 par le ministère de l'Enseignement Supérieur et de la Recherche sur son site : $\mathrm{http}: / /$ www.enseignementsuprecherche.gouv.fr/pid24447/taux-dinsertion-professionnelle-des-etudiants-par-universite-et-discipline.html
} 
domaines de formation, voire des spécialités détaillées, avec les emplois occupés.

En montrant que l'adéquation était loin d'être réalisée pour la plupart des spécialités de formation, les multiples recherches menées, depuis les années 70, ont mis en évidence que cette notion posait de nombreux problèmes et, notamment, ne pouvait pas jouer le rôle que tient la correspondance entre niveaux dans les études sur la suréducation (Giret, Lopez, Rose, 2005 ; Béduwé, Giret, 2010). En effet, le postulat que le seul bon appariement («match») est celui qui réalise la correspondance entre la spécialité de formation et la spécialité d'emploi, repose sur une vision erronée du marché du travail, celle de sous-marchés totalement isolés avec peu de substitutions possibles entre les formations pour accéder à un emploi donné. Dès lors, le constat de l'inadéquation entre spécialité de formation et spécialité d'emploi (y compris entre groupes de spécialités), s'il est éventuellement un élément de l'analyse, ne peut pas servir d'indicateur d'évaluation et de révélateur des problèmes posés par l'insertion des sortants de chaque formation.

Il est pourtant nécessaire, dans une démarche d'évaluation d'une formation, de connaître les emplois occupés par les jeunes et d'expliquer le rôle de leur formation dans l'accès à ces emplois. L'approche que nous proposons est fondée sur la notion de concentration versus dispersion des débouchés à l'issue d'une formation. Nous avons utilisé l'indice de concentration Gini, à l'instar de Dumartin 1997 ; Heijke et alii 2003 ; Chardon 2005, mais d'autres ont utilisé le nombre de professions concentrant $75 \%$ des jeunes d'une formation (Giret, Moullet, 2008) ou la formation la mieux représentée dans le recrutement d'un emploi (Affichard, Combes \& Grelet, 1992).

L'indice de concentration part, pour chaque formation, de l'observation des emplois réellement occupés par les jeunes et de la fréquence des embauches sur chacun de ces emplois au cours de la période d'insertion (Béduwé, Espinasse, Vincens, 2007). On parle ainsi d'approche statistique, parce qu'à aucun moment on ne préjuge de la qualité des emplois occupés (ni en niveau, ni en spécialité). L'indice de concentration varie entre 1 , cas où tous les jeunes d'une formation donnée ont pris le même emploi (au sens de la nomenclature Professions et Catégories Sociales de
l'Insee) et $0 \mathrm{si}$, au contraire, les jeunes ont tous pris des emplois différents ou sont également répartis entre plusieurs emplois.

L'indice de concentration considère deux éléments qui nous semblent essentiels pour évaluer l'insertion des sortants de chaque formation. Il tient compte des emplois réellement occupés sans porter de jugement normatif relatif à un bon ou mauvais appariement, et, comme on va s'attacher à le montrer, il permet de tenir compte des concurrences entre formations, c'est-àdire entre formés, sur le marché du travail. Ce faisant, il permet de mieux comprendre le rôle propre de la formation dans les résultats de l'insertion professionnelle. L'explicitation de ce rôle est un élément incontournable de l'évaluation.

La première partie de l'article va montrer que la concentration des débouchés s'explique par une analyse du marché du travail fondée sur la décomposition des formations et des emplois en types de compétences. Elle débouche sur la notion d'avantage compétitif attribué aux différentes formations. On montre alors, en deuxième partie, que les principaux résultats de l'insertion (salaire, chômage, utilisation des compétences, satisfaction) sont effectivement liés à la concentration.

\section{POURQUOI UNE PLUS OU MOINS GRANDE CONCENTRATION DES DÉBOUCHÉS À L'ISSUE D'UNE FORMATION?}

Pour étudier la concentration, il faut d'abord savoir si les principales théories du marché du travail fournissent des éléments d'analyse pertinents. Il ressort qu'une typologie appropriée des compétences fournies par les formations ou requises par les emplois est nécessaire. Cette typologie permet de cerner la notion d'avantage compétitif d'une formation pour certains emplois et donc de préciser le jeu de la concurrence entre les sortants. Cela conduit à formuler quelques hypothèses sur les relations entre le degré de concentration des débouchés et certains autres indicateurs de l'insertion professionnelle. 


\section{Des théories pour éclairer la diversité des emplois occupés}

Chaque théorie économique est une représentation du marché du travail. Il n'est pas étonnant que chacune « se pose en s'opposant », c'est-à-dire accentue sa différence avec les autres. Aucune ne semble évoquer explicitement la question de la concentration, mais chacune fournit les éléments d'une explication. Trois théories sont brièvement analysées : la théorie du capital humain, celle de la concurrence pour l'emploi et enfin la théorie de l'allocation (« assignment »).

\section{La théorie du capital humain}

Pour cette théorie, la formation confère une productivité potentielle qui se concrétise si l'individu parvient à utiliser ses compétences. Cette théorie soutient que les employeurs sont toujours conduits à proposer l'emploi qui utilise les compétences individuelles de la façon la plus efficace. Il en est ainsi, selon elle, pour deux raisons :

La première est que les salaires sont flexibles et que s'il y a un excès d'offre de certaines compétences, le salaire baissera (augmentera dans le cas contraire) ; comme le volume de la demande de travail est déterminé par l'égalité entre le produit marginal et le salaire fixé par le marché, une baisse du salaire provoque une hausse de la demande de travail ; un nouvel équilibre tend à s'instaurer.

La seconde raison est que la demande de chaque type de compétences est sensible aux variations de salaire. En effet, les entreprises peuvent aisément réorganiser la division du travail pour obtenir le même produit global avec des combinaisons différentes de compétences.

Cette représentation du marché du travail et de l'organisation de l'entreprise permet de dire, en un raccourci ambigu, que l'emploi (au sens de «job») n'apparaît pas dans la théorie du capital humain et que la productivité est attachée à l'individu. En fait, l'emploi existe, mais les hypothèses précédentes aboutissent à la conclusion que le volume global et le volume de chaque type d'emploi dépendent de l'offre de travail, en raison notamment de la plasticité du contenu des emplois et de leurs agencements dans le cadre de la division du travail.

S'agissant des différences de salaire selon les formations, la réponse de la théorie est simple. Si deux formations exigent le même investissement en capital humain, à l'équilibre, les salaires doivent être égaux. La théorie exclut les déclassements, sauf provisoires (ou apparents parce que la même durée de formation ne traduirait pas en réalité le même volume d'acquisition de compétences).

Dès lors, la différence de concentration pour des formations de même niveau pourrait s'expliquer de deux façons. (i) La première se référerait explicitement ou non à l'existence de sous-marchés du travail, chaque formation ayant une cible professionnelle précise. Autrement dit, les compétences de l'individu ne pourraient être pleinement utilisées que dans une famille d'emplois bien déterminée ; si le marché du travail ne permet pas un équilibre instantané, les individus issus de cette formation peuvent prendre provisoirement des emplois déclassés et moins rémunérés ; la concentration sera faible. En revanche, si les formés ont pu trouver les emplois correspondant à leurs compétences parce que les employeurs ont réagi rapidement à l'offre, la concentration sera forte. (ii) Selon la seconde explication, si deux formations de même niveau ont des concentrations différentes, cela signifie que la gamme d'emplois où leurs compétences sont pleinement utilisées est plus large pour l'une que pour l'autre ; celle qui est la moins concentrée concerne un plus large domaine d'utilisation; à l'équilibre, les deux groupes de formés occupent des emplois conformes à leurs compétences. Le salaire moyen doit-il être le même dans les deux groupes ? Pour répondre à cette question, il faut savoir pourquoi une formation procure des compétences dont le domaine d'utilisation est plus large que l'autre. S'il existe des compétences propres à certains emplois et que les formations qui visent ces emplois limitent de ce fait le champ d'utilisation des compétences qu'elles procurent, on pourrait s'attendre à une différence de salaire en faveur de ces formations; en quelque sorte, les individus auraient accepté de limiter le domaine d'utilisation de leurs compétences contre la perspective d'un salaire plus élevé compensant le risque dû à la plus grande étroitesse de leur marché ; il s'agirait d'un cas où « les différences sont égalisatrices », selon 
le schéma bien connu; mais alors le même volume de capital humain conduirait à une rémunération différente.

En définitive, cependant, il ne semble pas nécessaire de s'appesantir sur la théorie du capital humain dans le cadre du problème posé. En effet, une hypothèse fondamentale sur laquelle elle s'appuie reste celle de la flexibilité des salaires. Or, les tenants de la théorie déplorent que les marchés du travail soient affectés par leur trop grande rigidité. Si les salaires sont rigides, il n'y a aucune raison de s'attendre à ce que les prédictions de la théorie soient confirmées par les faits.

\section{La théorie de la concurrence pour l'emploi}

Cette théorie, proposée par Thurow (1975), utilise quelques éléments de la théorie du filtre ou du signal et de la théorie des marchés internes. La productivité est liée à l'emploi ainsi que le salaire. Les compétences nécessaires pour occuper efficacement l'emploi sont en grande partie acquises sur le tas. L'employeur doit donc chercher l'individu qui pourra acquérir ces compétences au moindre coût. La formation sert de filtre. Pour simplifier, Thurow ne retient que le niveau de la formation, mais il est évident pour lui que son contenu joue un rôle. Plus les compétences à acquérir sur le tas sont importantes et complexes et plus le niveau de recrutement devra être élevé. Les individus sont ainsi placés dans une file d'attente et le rang de chacun dépend de son niveau de formation. Ainsi, les individus sont incités à élever leur niveau de formation pour gagner des places dans la file d'attente. On retrouve la théorie du signal.

Dans le cadre de ce modèle, comment expliquer les différences de concentration des débouchés selon les formations? Les sortants de chaque formation prendraient place dans les files d'attente d'un certain nombre d'emplois. La position dans la file d'attente d'un emploi donné dépendrait du niveau et du contenu de la formation. Les sortants d'une formation qui se trouveraient en tête de la file d'attente pour un petit nombre d'emplois auraient une forte probabilité d'être recrutés et la concentration serait forte pourvu que la demande soit suffisante.

Mais les conditions permettant à ces individus d'être en tête de quelques files d'attente ne sont pas claires, surtout si on accepte l'hypothèse fondamentale que l'essentiel des compétences requises par l'emploi est acquis sur le tas. On se trouve en effet devant le dilemme suivant : (i) ou bien le contenu de la formation a peu d'importance pour acquérir les compétences acquises sur le tas et le niveau de formation joue alors le rôle essentiel. Les sortants d'une formation déterminée sont en concurrence avec les sortants de la plupart des formations de même niveau pour la place sur les listes d'attente d'un grand nombre d'emplois et la concentration n'a aucune raison d'être élevée ; (ii) ou bien le contenu de la formation joue un rôle important à côté du niveau de formation pour la place sur la liste d'attente d'un emploi donné, mais alors cela signifie probablement qu'une grande partie des compétences requises par l'emploi peuvent être acquises hors de l'emploi, ce qui est contraire à l'hypothèse de départ.

\section{La théorie de l'allocation (" assignment »).}

On se réfère ici surtout aux travaux de Sattinger (1993). Cette théorie se situe, selon Mc Guinness (2006), entre les deux précédentes théories. Elle s'oppose à la théorie du capital humain en considérant que la plasticité des contenus et des structures d'emplois est limitée à court terme ; dès lors, il n'y a aucune raison que tous les individus puissent occuper l'emploi « fait sur mesure » pour utiliser toutes leurs compétences. Mais elle s'éloigne de la théorie de la concurrence pour l'emploi en soutenant que l'allocation des individus aux emplois n'est pas une simple loterie ; les caractéristiques des individus influencent sa productivité dans un emploi donné et donc vraisemblablement son salaire. Le choix de l'employeur résulte d'un calcul efficience/coût ; ce calcul comportant le salaire et le coût d'acquisition des compétences qui font défaut au moment du recrutement. La théorie a pour objet essentiel d'expliquer les changements dans la distribution des gains en considérant à la fois les caractéristiques des individus et des emplois. Quant à la plus ou moins grande concentration des débouchés d'une formation, il est difficile de déduire de la théorie une explication immédiate. Mais l'idée centrale que l'accès à l'emploi dépend des caractéristiques de l'emploi et de l'individu sera un des éléments de l'explication que nous allons proposer. 


\section{Une typologie des compétences pour l'analyse de la relation formation-emploi}

En s'appuyant sur la typologie établie par C. Stasz, on propose de caractériser les formations et on montre ensuite que ces distinctions permettent aussi de caractériser les emplois.

\section{Caractériser les formations par leurs différences ou leurs ressemblances}

La distinction classique entre formation générale et formation spécifique à l'entreprise a été transposée aux emplois et non aux entreprises. Elle a conduit à distinguer les compétences générales qui peuvent se retrouver dans tous les emplois et les compétences spécifiques à un emploi qui ne sont utiles que dans celui-ci ${ }^{3}$.

Dans une perspective différente, C. Stasz (2001) estime qu'on peut identifier, dans la littérature spécialisée, quatre grands domaines de compétences (skills) :

- Les compétences académiques ou cognitives, le plus souvent associées à des disciplines scientifiques ;

- Les compétences «génériques», capacité à résoudre des problèmes, à communiquer, à travailler en équipe ;

- Les compétences techniques qui renvoient aux compétences nécessaires dans un emploi. Elles sont souvent codifiées dans les descriptions d'emplois ;

- Les attitudes ou «soft skills» $»^{4}$, les plus difficiles à définir. Stasz écrit : «le terme peut être compris en se référant à des notions comme celles de motivation, de volition et de dispositions $»^{5}$.

Ces quatre types de compétences se combinent dans ce que le même auteur appelle «l'approche économique » des compétences qui considère celles-ci comme des attributs de l'individu. Une formation

\footnotetext{
${ }^{3}$ Pour une application récente de cette distinction, voir Bruyère et Lemistre (2010)

${ }^{4}$ Les compétences dites douces sont orientées vers les interactions humaines et font appel à l'intelligence émotionnelle (communication, négociation, empathie, gestion des conflits, du temps, etc.). Elles sont complémentaires à la formation académique ou technique. Mais pour certains auteurs, les soft skills joueraient un rôle croissant sur le marché du travail.

5 «the term can refer to such constructs as motivation, volition and dispositions ».
}

procure essentiellement des compétences des trois premières catégories, mais certains cursus de formation peuvent s'attacher à développer la quatrième.

Dès lors, on peut tenter de caractériser les formations en s'attachant à ce qui les rapproche ou les différencie. Une formation procure trois types de compétences :

- Des compétences générales qui comprennent essentiellement des compétences cognitives et génériques et qui sont très liées au niveau de la formation. En simplifiant, on pourrait admettre que toutes les formations d'un niveau donné permettent d'acquérir les mêmes compétences générales.

- Des compétences partagées, c'est-à-dire des compétences cognitives, techniques et génériques qui se retrouvent dans plusieurs formations ; par exemple, toutes les formations scientifiques de niveau bac +5 ont un « socle minimum » commun en mathématiques.

- Des compétences propres à la formation, c'est-àdire qui ne sont fournies que par elle et qui le plus souvent sont à l'origine de son appellation. Il s'agit surtout de compétences cognitives et techniques, peutêtre aussi d'attitudes.

La spécificité d'une formation dépend évidemment de la combinaison de ces compétences et non de la seule addition d'éléments cognitifs, génériques ou techniques.

\section{Les emplois : des compétences requises aux compétences présumées}

Du côté des emplois, l'analyse des compétences requises peut aussi concerner les formations puisque le problème est ici celui du recrutement de débutants. Le point essentiel tient à la grande variété des cas possibles. Certains emplois exigeront surtout des compétences générales ou des compétences partagées, les compétences spécifiques à ces emplois pouvant être acquises rapidement après le recrutement. Pour ces emplois, les entreprises peuvent recruter des jeunes sortants de plusieurs formations, toutes celles qui procureront ces compétences générales ou partagées. D'autres emplois exigeront au contraire des compétences spécifiques importantes qui exigent un long apprentissage ; si celui-ci est fourni par une formation extérieure aux entreprises, il occupera la plus grande partie du temps consacré à ce cursus de formation ; la 
part des compétences partagées sera faible. Ainsi, la distinction entre formations professionnelles et formations générales ne permet pas de capter l'essentiel de ce qui fonde la concurrence pour les emplois. En effet, plusieurs formations dites professionnelles peuvent viser des emplois, certes différents mais tous caractérisés par la place tenue par des compétences partagées présentes dans toutes les formations concernées. Les compétences spécifiques à chacun de ces emplois sont certes acquises grâce à une formation et une seule, la formation professionnelle conçue pour cet emploi, mais la possibilité d'acquérir à un coût assez faible ces compétences spécifiques permet à l'entreprise d'élargir le champ des recrutements possibles.

Ainsi, pour un employeur qui souhaite pourvoir un emploi en s'adressant à un débutant, la question est «quelles sont les formations où il est possible de trouver des candidats possédant l'essentiel des compétences dont j'ai besoin ? ». De même, le diplôme attestant sa formation permet à un individu de se poser la question (à supposer qu'il n'y ait pas encore réfléchi...) « avec cette formation, à quels emplois puis-je postuler? ». Les réponses à ce double questionnement dépendent des comparaisons entre compétences présumées des individus et compétences requises par les emplois. Les relations entre les deux ensembles, individus et emplois, sont ainsi structurées par des concurrences très diversifiées qui déterminent le degré de concentration.

\section{L'avantage compétitif, expression des possibilités de concurrence}

La concurrence peut être envisagée au regard d'un emploi donné ou du point de vue des individus en quête d'emploi.

\section{Au regard d'un emploi donné}

Ici, les sortants des différentes formations ont un avantage compétitif qui est nul si la candidature n'a aucune chance d'être examinée (on dira que l'individu n'est pas éligible à cet emploi), et qui peut être plus ou moins important. Cet avantage compétitif est attribué par l'employeur'.

${ }^{6}$ Mais il peut dépendre aussi de l'efficacité du marketing réalisé par la formation auprès des employeurs futurs.
Pour le sortant d'une formation donnée, il se traduit par la probabilité d'être recruté si un de ces emplois est vacant. Plusieurs formations peuvent avoir le même avantage à l'égard d'un emploi; il est probable que, dans ce cas, ce sont leurs compétences partagées qui vont leur procurer cet avantage. Lorsque l'emploi exige d'importantes compétences spécifiques qui ne sont fournies que par une seule formation, l'avantage compétitif de celle-ci par rapport à toutes les autres est très grand ; il est absolu dans le cas des emplois réglementés où un diplôme d'exercice est requis.

Les notions d'éligibilité et d'avantage compétitif s'appuient, d'une part, sur la notion de recouvrement partiel des contenus des formations et, d'autre part, sur les différences entre les types de compétences demandées par les emplois. Elles permettent d'abandonner la représentation des relations formationsemplois comme un jeu où des pièces de différentes formes doivent être placées dans les cases correspondantes. Elles suggèrent une autre représentation où les pièces et les cases ne sont pas totalement rigides et où les coûts d'adaptation et d'apprentissage des individus sont relativement faibles (Stankiewicz, 1998).

\section{Du côté des individus entrant sur le marché du travail}

La formation des individus est un indicateur des types d'emplois qu'ils peuvent chercher. Ainsi l'horizon professionnel des sortants d'une formation est structuré par le jeu des avantages compétitifs qu'ils peuvent posséder du fait de leur formation. Ils connaissent probablement, au moins approximativement, les avantages compétitifs dont ils disposent pour différents emplois ${ }^{7}$; dès lors, compte tenu de leurs préférences et de leurs contraintes personnelles, ils cherchent en priorité ceux qu'ils estiment les meilleurs en fonction de l'état du marché. L'influence de la formation sur l'insertion s'exerce donc par l'intermédiaire des avantages compétitifs.

Quand une formation procure un fort avantage compétitif pour un ou deux emplois et aucun pour les autres emplois, il est probable que la plupart des sortants de cette formation vont chercher en priorité les emplois

\footnotetext{
7 Parmi lesquels, évidemment, les emplois dans la cible des formations professionnelles.
} 
pour lesquels ils possèdent cet avantage. Lorsque cette recherche réussit, les sortants de cette formation ont tendance à se retrouver dans ces quelques emplois; la concentration des débouchés sera donc élevée. Lorsque la recherche échoue en raison de l'insuffisance de la demande, certains, voire aucun, des sortants de la formation ne pourront concrétiser leur avantage compétitif. S'ils ne souhaitent pas poursuivre leur recherche, ils se répartiront entre les autres emplois auxquels ils sont éligibles en fonction de leurs préférences ou contraintes et de l'état du marché. L'indice de concentration de la formation sera moins élevé, voire faible. Quand une formation, au contraire, ne procure d'avantage compétitif pour aucun emploi, les sortants se tourneront vers des emplois largement ouverts à la concurrence, selon les occasions offertes par le marché. L'indice de concentration sera faible.

\section{L'indice de concentration, un indicateur de l'impact de la formation}

La concentration des débouchés d'une formation va donc dépendre de ses avantages compétitifs et de l'état du marché du travail. Une formation dont l'indice de concentration est élevé présente un avantage compétitif pour quelques emplois et la majorité des formés a pu le faire valoir. Une formation dont l'indice est faible présente moins d'avantage compétitif ou alors les jeunes n'ont pas pu les utiliser de manière probante. L'indice de concentration est donc un indicateur du positionnement concurrentiel de chaque formation à un moment donné d'observation.

Ainsi, l'indice de concentration constitue un indicateur de l'influence de la formation au cours de la période d'insertion. Cette influence s'exerce surtout sur la qualité des emplois obtenus plutôt que sur l'accès à l'emploi en général. Plus l'indice de concentration est élevé et plus on pourra affirmer que la formation a joué un rôle en permettant aux sortants, collectivement, via leur avantage compétitif, d'accéder à quelques emplois particuliers. Ils auront, collectivement, jugé ces quelques emplois préférables aux autres emplois qui leur étaient accessibles. À l'inverse, plus l'indice est faible et moins la formation aura exercé d'influence sur le choix des emplois. Que ce soit parce qu'ils n'avaient pas d'avantage compétitif pour des emplois particuliers, ou parce qu'ils ont éprouvé des difficultés à le faire valoir, le choix des emplois pris va surtout dépendre des préférences ou contraintes de chacun ainsi que des opportunités offertes par le marché du travail.

Ceci nous conduit à formuler quatre hypothèses sur le lien entre la concentration des débouchés d'une formation et ses résultats d'insertion :

Hypothèse 1 : La relation entre indice de concentration et chômage est difficile à prévoir. En effet, la concentration des débouchés signifie que les jeunes ont pu faire valoir l'avantage compétitif attribué à leur formation. Dès lors, le chômage sera faible. Mais la recherche d'emploi peut prendre du temps et les jeunes peuvent soit rester au chômage, soit accepter des emplois d'attente. La combinaison de ces effets contradictoires suggère que la relation négative entre chômage et concentration doit être faible.

Hypothèse 2 : Quels que soient les emplois occupés, s'il y a concentration des sortants d'une formation dans quelques emplois, cela signifie que ces individus les ont préférés aux autres emplois accessibles; et parmi les motifs de cette préférence, la rémunération joue sans doute un rôle. Sortir d'une formation aux débouchés concentrés doit procurer un bonus salarial.

Hypothèse 3 : L'accès privilégié à quelques emplois, quels qu'ils soient, signifie que les individus possédaient un avantage compétitif et qu'ils l'ont fait valoir. Cet avantage compétitif, parce qu'il est collectif et partagé par l'ensemble des sortants de la formation, repose sur les compétences acquises en formation. On s'attend donc à ce que les jeunes sortants d'une formation aux débouchés concentrés accèdent plus souvent à des emplois où ils jugent leurs compétences correctement utilisées.

Hypothèse 4 : Quels que soient les emplois occupés, s'il y a concentration des sortants d'une formation dans quelques emplois, cela signifie que ces individus ont collectivement pu accéder aux emplois qu'ils jugeaient préférables pour eux. On s'attend donc à ce que les jeunes qui sortent d'une formation aux débouchés concentrés soient plus satisfaits de leur situation professionnelle et à ce qu'ils cherchent moins à changer d'emploi.

Ces quatre hypothèses vont être maintenant testées. 


\section{L'INDICE DE CONCENTRATION, POUR ANALYSER L'INSERTION PROFESSIONNELLE}

La première partie est consacrée aux données utilisées et à la construction de nos indicateurs de résultat sur l'insertion professionnelle. La deuxième porte sur la discussion de la première hypothèse en s'appuyant sur l'étude détaillée de quatre formations particulières. Enfin, la troisième partie porte sur le test des trois dernières hypothèses à l'aide de modèles sur données individuelles.

\section{Données sur l'insertion professionnelle}

Nous utilisons les données de l'enquête Génération 98 du Céreq. Nous avons choisi de restreindre l'analyse aux 30200 sortants de l'enseignement supérieur afin de travailler sur un champ plus homogène en termes de problématique d'insertion professionnelle. La démarche pourrait cependant être étendue aux formations secondaires.

L'enquête interroge des sortants de toutes les formations de l'enseignement supérieur. Ces formations sont repérées par le type de diplôme auquel elles conduisent (Diplôme d'École, Doctorat, DESS, DEA, DUT, BTS, $\mathrm{DEUG}^{8}$ ) et leur spécialité ${ }^{9}$. Pour toutes les formations, on distingue en plus les sorties avec réussite des sorties sur échec. En ne retenant que les formations dont l'effectif interrogé est supérieur à 30 individus, on distingue 159 formations différentes ${ }^{10}$. Les jeunes sortis de ces formations ont décrit leurs trois premières années de vie professionnelle, et notamment tous les emplois qu'ils ont occupés entre 1998 et 2001. On connaît donc l'éventail des emplois, codés à l'aide de la nomenclature PCS de l'INSEE, pris à l'issue de chacune de ces formations et avec quelle fréquence. Ces deux informations sont à la base du calcul de

${ }^{8}$ Diplôme d'études supérieures spécialisées, Diplôme d'études approfondies, Diplôme universitaire de technologie, Brevet de technicien supérieur, Diplôme d'études universitaires générales.

${ }^{9}$ Issue d'un regroupement du Code de la Nomenclature des Spécialités de Formation (NSF) à 4 chiffres opéré par le Céreq au moment de la constitution de la base de sondage de l'enquête.

${ }^{10}$ Voir Béduwé et Vincens (2010), pour la liste complète des formations. l'indice de concentration des débouchés d'une formation. À partir des réponses individuelles des jeunes aux questions concernant le déroulement de leur insertion professionnelle, on peut également calculer des indicateurs de résultat pour chaque formation.

Nos quatre hypothèses reposent sur la situation des jeunes vis-à-vis des critères de chômage (H1), de rémunération (H2), d'utilisation de leurs compétences (H3), et de satisfaction (H4). Les indicateurs de résultat qui en découlent sont construits de la manière suivante :

- Situation vis-à-vis du chômage : nombre de mois de chômage en trois ans ;

- Rémunération: salaire relatif (moyenne des salaires obtenus par les formés au cours des trois ans, rapportée au salaire moyen des formations de même niveau);

- Satisfaction quant aux compétences utilisées : la proportion de jeunes qui déclarent être utilisés à leur niveau de compétences ( $v . s$ en-dessous ou au-dessus) dans l'emploi qu'ils occupent ;

- Satisfaction quant à la situation professionnelle : taux de jeunes qui, au moment de l'enquête en 2001, qu'ils soient en emploi ou non, déclarent que leur situation leur convient ;

- Recherche d'un autre emploi : proportion de jeunes en emploi en 2001 déclarant rechercher un autre emploi.

Ces critères vont être corrélés avec l'indice de concentration (indice de Gini) des débouchés de chaque formation. Il est calculé comme suit :

$$
G f=\frac{1}{2 \mu n(n-1)} \sum_{i} \sum_{j}\left|x_{i}-x_{j}\right|, i=1-n, \text { où } n \text { est le }
$$

nombre total de catégories d'emplois, $x i$ est le nombre de jeunes diplômés de $f$ qui ont accédé à l'emploi $i$ parmi les $n$ possibles et $\mu$ le nombre moyen d'emplois pris par les jeunes ayant la formation / diplôme $f$.

L'indice de Gini rend compte à la fois du nombre d'emplois différents (i.e des PCS différentes) pris par les formés (dispersion) et de la fréquence des embauches sur chacun de ces emplois (modes). Le calcul se fait, pour chaque formation, sur l'ensemble des emplois pris par les jeunes au cours des trois ans d'observation. Il est compris entre 0 (cas où tous les sortants auraient pris des emplois différents ou seraient 
également répartis sur un nombre donné d'emplois) et 1 (cas où tous les sortants auraient pris le même emploi). Pour les formations supérieures dans l'enquête Génération 98, l'indice varie entre 0.76 et 0.99 .

Enfin, pour compléter l'analyse, on a retenu également les critères/indicateurs suivants :

- Stabilité de l'emploi (taux de CDI - contrat à durée indéterminée - à l'issue de la formation); part des embauches sur contrat à durée indéterminée, CDI de droit privé ou fonctionnaire ;

- Classement de l'emploi (taux d'emploi de bon niveau à l'issue de la formation); le taux d'emploi dont le niveau est au moins égal à celui de la formation (Affichard 81), autrement dit les emplois bien classés ou non déclassés.

\section{Posséder un avantage compétitif n'exclut pas le chômage}

L'étude des quatre formations particulières qui suit a un double objectif : montrer l'opérationnalité de la notion de concentration pour l'analyse de l'insertion professionnelle des formés et introduire la discussion de l'hypothèse H1 qui postule un faible lien entre chômage et concentration.

\section{Étude de quatre formations particulières}

Nous avons choisi d'étudier la destinée des jeunes sortis de quatre formations qui s'opposent à la fois en termes de chômage et d'indice de concentration : les docteurs en sciences, les titulaires d'un BTS de tourisme, les titulaires d'un BTS d'agronomie agriculture et les jeunes sortis après un échec au Deug d'AES (Administration économique et sociale). Les jeunes des deux premières formations ont connu des débouchés très concentrés, ceux des deux dernières ont au contraire des emplois très dispersés. Leurs indices de concentration sont respectivement de $0.95,0.90,0.78$ et 0.77 (tableau 1).

Pour chaque formation, on a tracé l'histogramme des 25 premiers emplois obtenus par les jeunes, ordonnés en fonction de leurs fréquences ${ }^{11}$ ( $c f$. graphes 1 à 4). Sont également reportés sur ces histogrammes le quartile de la valeur des indicateurs définis précédemment ( $c f$. tableau 1). Ces valeurs sont ainsi plus facilement comparables d'une formation à l'autre : un quartile élevé (Q4) signifie que le taux observé se situe dans le quart supérieur et que les résultats sont très bons.

\footnotetext{
11 Le choix de ne présenter que les 25 premiers emplois de chaque formation est uniquement dicté par le souci de lisibilité des libellés d'emploi sur le graphique.
}

Tableau 1

Quatre exemples de formations

\begin{tabular}{|c|c|c|c|c|c|c|c|c|c|c|c|c|}
\hline & 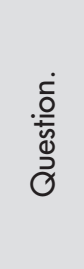 & 岇 & 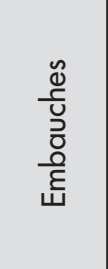 & 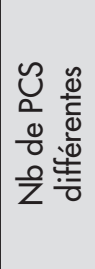 & 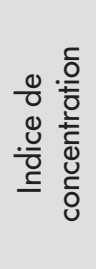 & 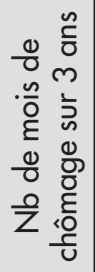 & 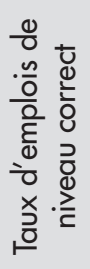 & 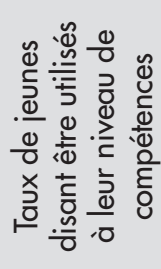 & 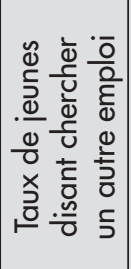 & 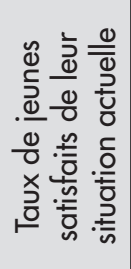 & 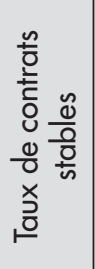 & 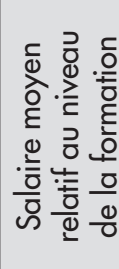 \\
\hline $\begin{array}{c}\text { Doctorat } \\
\text { de sciences }\end{array}$ & 714 & 2669 & 1202 & 86 & 0,95 & 3,8 & $88 \%$ & $82 \%$ & $23 \%$ & $75 \%$ & $39 \%$ & 1,04 \\
\hline $\begin{array}{c}\text { Échec au } \\
\text { Deug d'AES }\end{array}$ & 367 & 5228 & 707 & 133 & 0,78 & 5,4 & $25 \%$ & $50 \%$ & $36 \%$ & $65 \%$ & $24 \%$ & 0,96 \\
\hline $\begin{array}{c}\text { BTS } \\
\text { d'agronomie }\end{array}$ & 361 & 4984 & 768 & 142 & 0,77 & 2,3 & $32 \%$ & $60 \%$ & $17 \%$ & $75 \%$ & $32 \%$ & 0,89 \\
\hline $\begin{array}{c}\text { BTS hôtellerie } \\
\text { tourisme }\end{array}$ & 208 & 3016 & 480 & 79 & 0,90 & 2,3 & $28 \%$ & $59 \%$ & $28 \%$ & $68 \%$ & $40 \%$ & 0,89 \\
\hline
\end{tabular}

Source : Enquête Génération 98, formations de niveau supérieur, effectifs de sortants non pondérés $>30$.

Exemple de lecture : l'enquête contient 714 questionnaires de jeunes sortis avec un doctorat de sciences en 1998 (soit 2669 sortants estimés) pour lesquels on observe 1202 prises d'emploi en 3 ans sur 86 PCS différentes. Sur cette période, ils ont connu en moyenne 3,8 mois de chômage et 88 \% avaient un niveau d'emploi correspondant à celui de leur diplôme (i.e un emploi de Cadre). Leur salaire moyen était 1.04 fois supérieur à celui de l'ensemble des diplômés de niveau Doctorat. Le graphique 1 présente les recrutements opérés sur les 25 emplois les plus fréquents. 
Graphique 1

25 premiers emplois occupés après un Doctorat de Sciences entre 98 et 2001 (sur 86 )

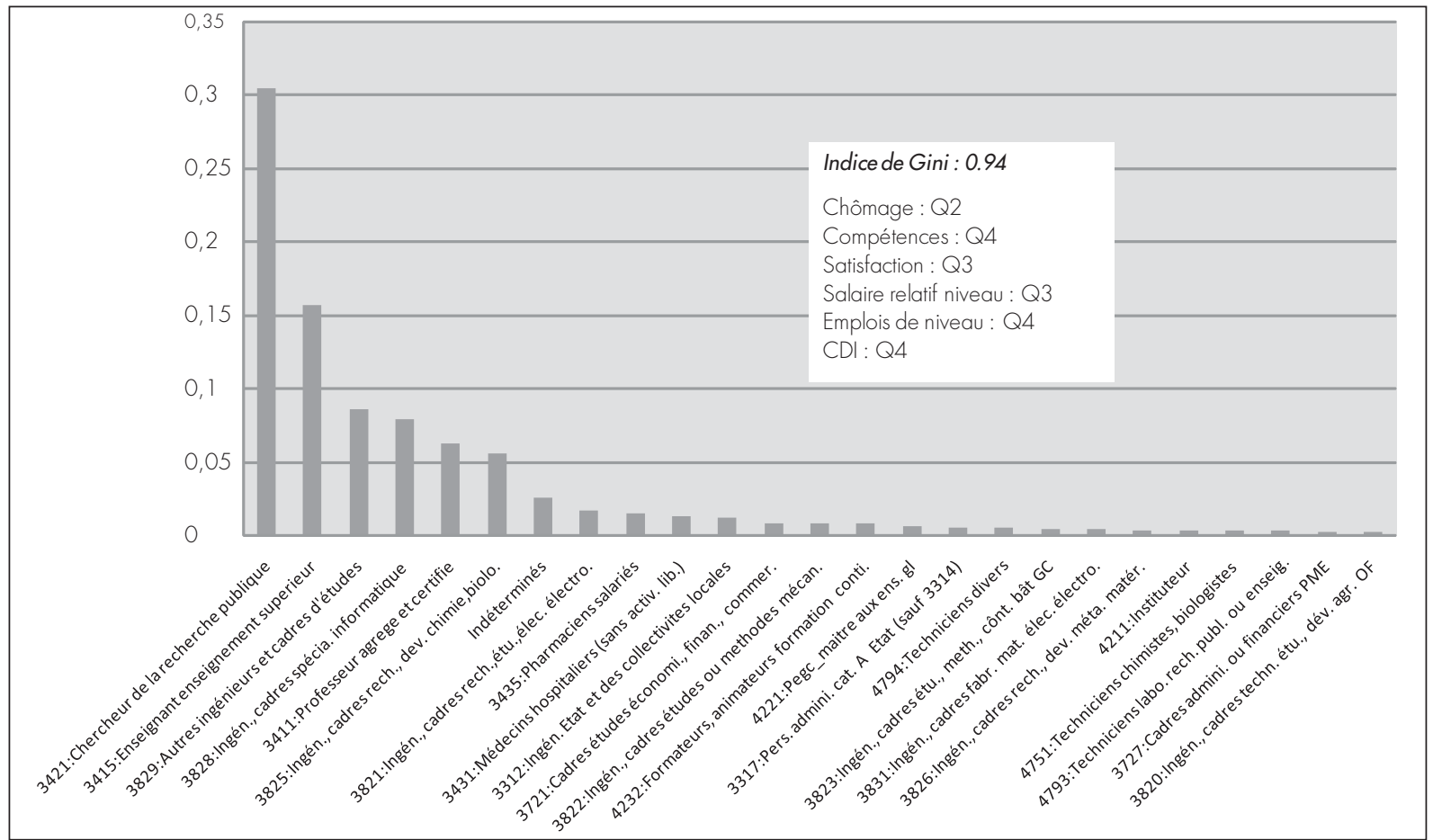

Note de lecture : Les diplômés d'un Doctorat de sciences ont eu 86 emplois différents. Le graphe représente les 25 premiers, classés par ordre de fréquence des recrutements. L'emploi de Chercheur de la recherche publique (PCS 3421) a totalisé $30.5 \%$ des embauches, celui d'Enseignant du supérieur (PCS 3415) 15,7\%, etc.

Graphique 2

\section{5 premiers emplois occupés après un BTS de Tourisme entre 98 et 2001 (sur 79)}

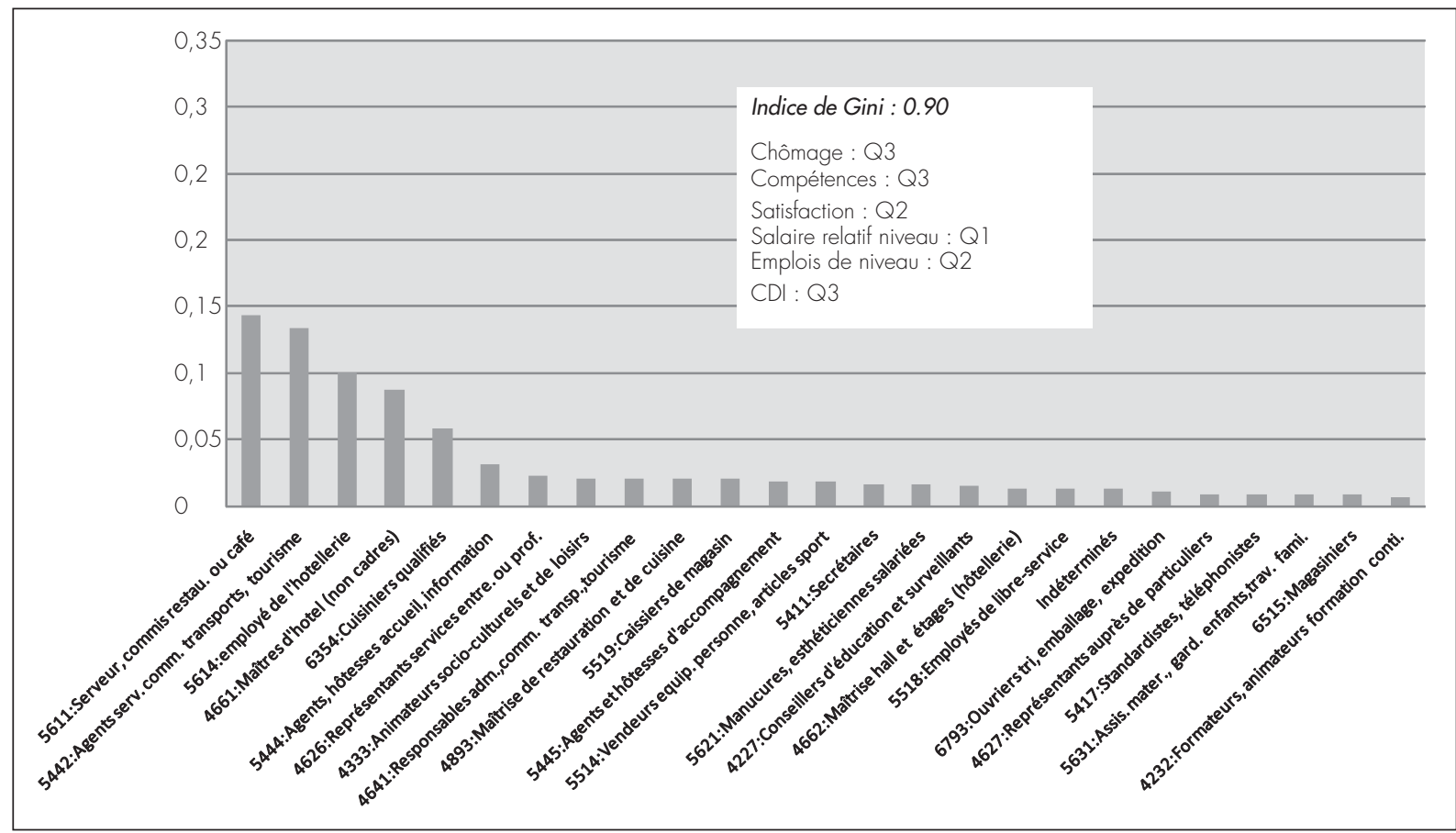


Graphique 3

25 premiers emplois occupés après un BTS Agronomie et Agriculture entre 98 et 2001 (sur 142)

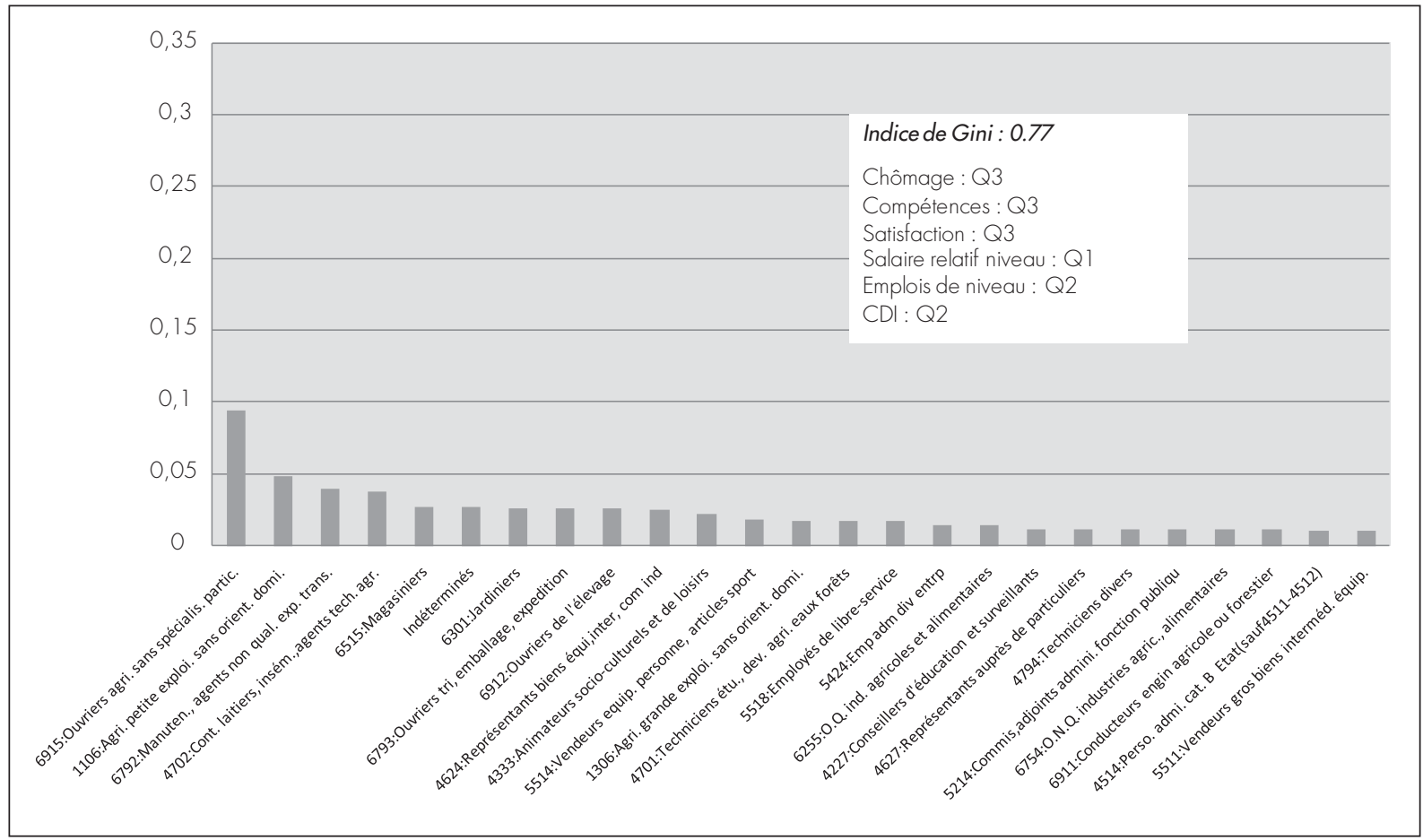

Graphique 4

25 premiers emplois occupés après une sortie sur échec au Deug d'AES entre 98 et 2001 (sur 133)

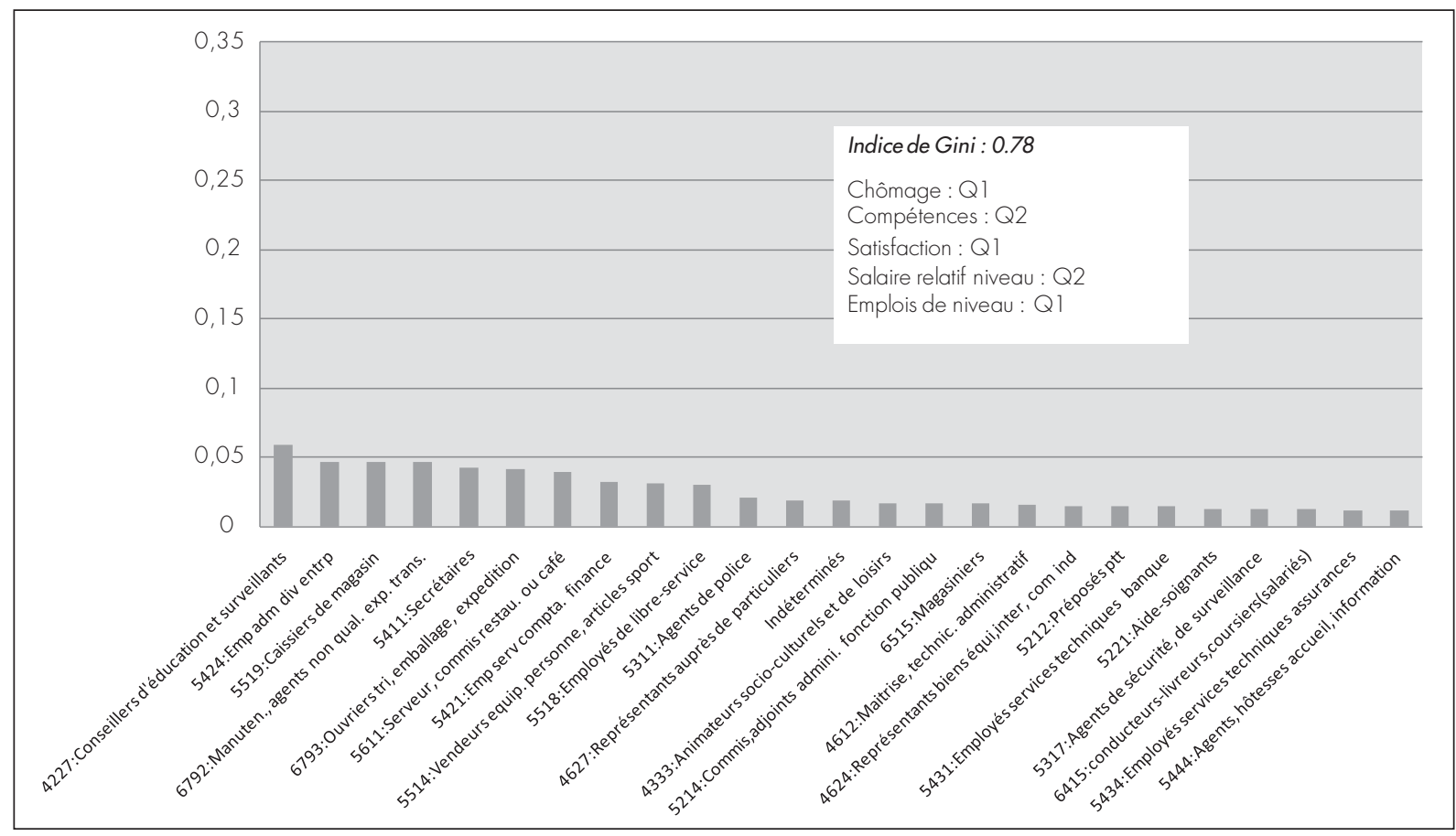

Source : Enquête Génération 98, Céreq. 
À l'inverse, le premier quartile (Q1) signifie que les résultats sont parmi les $25 \%$ les plus faibles.

Premier résultat (tableau 1): il existe une grande diversité d'emplois à l'issue de ces quatre formations (resp. 86, 133, 142 et 79). Ce résultat vaut pour l'ensemble des formations.

Cependant, et c'est le deuxième constat mis en évidence par les quatre graphiques, les Docteurs en sciences et les BTS de Tourisme sont, au-delà de cette diversité, concentrés sur 5-6 emplois, ce qui n'est pas le cas des deux autres formations. Ces 5-6 emplois concentrent à eux seuls resp. $75 \%$ et $52 \%$ des embauches. En comparaison, il faut 40, resp. 50 emplois différents pour totaliser $75 \%$ des embauches chez les échecs Deug d'AES, resp. les BTS d'agronomie. Ce deuxième constat est également général : quelle que soit la formation, lorsque son indice est élevé, la concentration se fait toujours sur quelques emplois seulement.

Enfin, troisième constat, les emplois qui présentent des fréquences importantes sont, quasiment toujours, en « relation avec le contenu de la formation». Ce résultat, que nous allons commenter pour les deux exemples de formations concentrées retenues, peut être généralisé après observation minutieuse des 159 formations.

Les six emplois qui «font» la concentration des Docteurs en sciences sont des empois qui correspondent bien à la cible de ces diplômes : chercheurs, enseignants chercheurs, ingénieurs. Les Docteurs en sciences ont un avantage compétitif évident pour les deux premiers emplois et important pour les emplois d'ingénieur, du fait de leurs compétences élargies voire spécifiques ${ }^{12}$. Ils sont en concurrence, pour ces emplois d'Ingénieur, avec d'autres diplômés, des diplômés d'école d'ingénieurs bien sûr, mais aussi par exemple des DESS de sciences ou d'informatique. Les emplois à faible effectif sont quasiment tous des emplois de niveau Cadre, ce qui confirme l'avantage compétitif de ces formations sur le marché du travail en général. Leur avantage compétitif pour ces emplois passe par les compétences partagées et/ ou générales acquises dans leur formation de Docteur.

12 Certains Docteurs, mais pas tous, possèdent également un diplôme d'Ingénieur.
Les autres indicateurs d'insertion, compétences, satisfaction, stabilité et salaire relatif de ce diplôme sont bons, voire très bons, à l'exception de l'indicateur de chômage qui est relativement élevé. Ces diplômés ont eu largement accès à de très bons emplois mais il leur a fallu, en moyenne, chercher un peu, faute de pouvoir accéder tous aux emplois de chercheur, d'enseignantchercheur ou d'ingénieur.

Les cinq ou six emplois concentrés des BTS Hôtellerie-Tourisme se situent dans le secteur de l'hôtellerie-restauration-tourisme. Ces diplômés ont eu un avantage compétitif évident pour ces emplois vers lesquels ils se sont collectivement dirigés, bien qu'ils soient tous déclassés par rapport à leur niveau de formation. Les emplois de bon niveau (Maîtrise et Cadres de l'hôtellerie) présentent de petites fréquences. Les jeunes se disent majoritairement utilisés à leur niveau de compétences, ce qui laisse penser qu'ils ne s'estiment pas déclassés. En revanche, ils sont peu satisfaits de leur situation, leurs salaires sont relativement bas et ils ont connu beaucoup d'emplois. On sait que les modes de gestion de ce secteur sont souvent mal vécus par les jeunes et entrainent un turn-over important. On observe également beaucoup d'emplois de petites fréquences sans lien apparent avec le contenu de leur formation. Tout ceci explique sans doute qu'ils n'aient in fine que peu souffert du chômage.

À l'inverse, les emplois pris à l'issue du BTS Agronomie sont extrêmement divers et rarement de bon niveau, mais se situent souvent dans l'agriculture ou l'agronomie. Leurs titulaires y sont vraisemblablement recrutés pour leurs compétences partagées ou générales, mais ils n'ont d'avantage compétitif pour aucun de ces emplois. Ainsi quelques jeunes sont agriculteur, technicien des Eaux et Forêts ou encore conducteur laitier, soit sur des emplois dont le niveau est en correspondance avec leur formation. Mais ces emplois font massivement appel à d'autres catégories de diplômés, notamment des Bac pro. Ils sont malgré tout six sur dix à se dire utilisés à leur niveau de compétence. Ces diplômés ont souvent changé d'emplois sans trop souffrir du chômage, ce qui peut expliquer qu'ils soient relativement satisfaits de leur situation.

Les sortants sur échec au Deug d'AES occupent, quant à eux, des emplois très dispersés et systématiquement 
non qualifiés. Tous les indicateurs d'insertion sont dans le rouge, y compris le chômage. Faute d'avantage compétitif pour aucun emploi, ces jeunes sont disséminés sur des emplois sans exigence vis-à-vis de la formation.

Cette analyse rapide monte l'intérêt de la notion de concentration pour évaluer le lien aux emplois de ces formations et expliquer la valeur des autres indicateurs. On peut la considérer comme une mise en cohérence des informations statistiques collectées et une introduction possible à des monographies faisant appel à d'autres sources d'analyse.

\section{Discussion sur la relation entre concentration et chômage (H1)}

Sur l'ensemble des formations supérieures, l'indice de Gini est faiblement mais négativement corrélé avec l'indicateur de chômage (-0.29). Ainsi, lorsque les débouchés sont concentrés, les jeunes ont éprouvé, en moyenne, assez peu de difficultés à trouver ces quelques emplois, mais la corrélation est faible. Cette faible corrélation vient de ce que les jeunes entrants sur le marché du travail vont opérer des arbitrages personnels entre recherche d'emploi et emploi satisfaisant. Ces arbitrages vont dépendre de leurs préférences et de leurs contraintes personnelles, du contexte économique, et, bien sur, de l'avantage compétitif qu'ils tirent de leur formation.

Une étude minutieuse de toutes les formations, selon leur niveau de concentration et de chômage, montre, effectivement, que les formations sont assez également réparties en quatre catégories : concentration élevée et chômage fort ou faible, concentration faible et chômage fort ou faible. Les formations choisies au paragraphe précédent sont typiques de ces quatre cas de comportement.

Les formations qui présentent des taux de chômage faibles et des indices de concentration élevés sont prestigieuses ou réglementées (Écoles d'ingénieurs, formations de la santé). Avantage spécifique et demande régulée se conjuguent. Mais lorsque la formation possède un avantage compétitif pour quelques emplois et que la demande sur ces emplois est - conjoncturellement - faible, les jeunes peuvent décider de consacrer du temps à chercher le bon emploi et les durées de chômage vont augmenter. C'est le cas des Docteurs en sciences, des diplômés en psychologie ou des domaines artistiques. La concentration peut donc s'accompagner ou non de chômage élevé.

De même, une grande dispersion des emplois peut se conjuguer ou non à un chômage élevé. Dans un cas, les jeunes trouvent rapidement du travail en faisant valoir les compétences générales ou partagées de leur formation. Leurs emplois sont dispersés mais ils ne connaissent que peu de chômage (il s'agit souvent de BTS, notamment non diplômés). Dans l'autre cas, faute d'avantage compétitif quelconque et donc en position de concurrence très défavorable sur le marché du travail, les jeunes mettent du temps à trouver des emplois ; ceux-ci étant dispersés et, dans ce cas, souvent peu ou pas qualifiés (c'est le cas de pratiquement tous les échecs au Deug). La coexistence de ces quatre cas valide, empiriquement, notre première hypothèse : en raison de la diversité des préférences/ contraintes individuelles des jeunes au moment de prendre leurs premiers emplois, la concentration des débouchés ne s'accompagne pas toujours d'un chômage faible.

\section{Posséder un avantage compétitif augmente les chances d'accès aux meilleurs emplois}

La notion de meilleur emploi ou meilleure situation professionnelle renvoie respectivement à celles de meilleure rémunération $(\mathrm{H} 2)$, meilleure utilisation des compétences $(\mathrm{H} 3)$ et meilleure satisfaction. Les trois hypothèses sont d'abord testées au niveau de la formation et ensuite au niveau des individus de chaque formation à l'aide de modèles sur données individuelles.

\section{Indicateurs d'insertion et statistiques descriptives}

Nos trois dernières hypothèses supposent l'existence d'un lien entre certains indicateurs de résultat d'insertion et l'indice de concentration des formations. Les corrélations données dans le tableau 2 en sont une première validation. Plus les débouchés à l'issue d'une formation sont concentrés et plus les salaires sont, en moyenne, élevés (H2), plus les jeunes se disent utilisés à leur niveau de compétence (H3), 
satisfaits de leur situation et moins à la recherche d'un autre emploi (H4).

Ces quatre indicateurs sont également liés à trois autres indicateurs : taux d'emplois de bon niveau, taux d'emplois stables et nombre de mois de chômage.

Ces corrélations sont souvent relativement faibles : une bonne rémunération ou une situation jugée satisfaisante ne signifie pas forcément l'accès rapide, à des emplois stables et bien classés pour tous. Si les jeunes avaient tous effectué les mêmes « choix », ces corrélations seraient plus élevées et la réussite de l'insertion s'apprécierait indifféremment par l'un ou l'autre de ces critères.

Mais ces corrélations ne sont pas non plus nulles car, bien évidemment, certains jeunes, issus de formations très « performantes », tirent leur épingle du jeu sur la plupart des critères. Ainsi, le taux de satisfaction augmente lorsque le chômage à l'issue de la formation est faible (-0.60), lorsque l'emploi est stable (0.46) et de bon niveau (0.51). De même, les jeunes sont plus nombreux à se dire utilisés à leur niveau de compétences lorsqu'ils ont trouvé rapidement du travail (-0.50), en CDI (0.59) ou, ce qui parait plus évident, sur des emplois correspondant à leur niveau de formation (0.71).
Il convient donc, pour tester nos hypothèses, de départager l'effet de concentration des effets de chômage, de déclassement et/ou de stabilité sur nos quatre indicateurs d'insertion. Nous mobilisons donc des analyses prenant en compte les comportements individuels des jeunes au moment de leur recherche d'emploi.

\section{Test des hypothèses $\mathrm{H} 2, \mathrm{H} 3$ et $\mathrm{H} 4$}

Plus les débouchés à l'issue d'une formation sont concentrés et plus on s'attend à ce que les salaires soient, en moyenne, élevés (H2), à ce que les jeunes se disent utilisés à leur niveau de compétence (H3), satisfaits de leur situation et moins à la recherche d'un autre emploi (H4).

Ces trois hypothèses reposent sur l'idée que, plus la formation a des débouchés concentrés, plus les jeunes ont pu faire valoir - collectivement - l'avantage compétitif accordé à leur formation et accéder collectivement - aux meilleurs emplois parmi tous ceux qui leur étaient accessibles. Pour les tester, quatre modélisations sur données individuelles sont utilisées (tableau 2): Une régression linéaire où la variable à expliquer est le logarithme du salaire mensuel (H2, col. 1), des modèles de type logit où les variables indépendantes sont la réponse des jeunes à

Tableau 2

Corrélations entre indicateurs d'insertion et indice de Gini

\begin{tabular}{|l|c|c|c|c|}
\cline { 2 - 5 } \multicolumn{1}{c|}{} & $\begin{array}{c}\text { H2: } \\
\text { Rémunération } \\
\text { (salaire moyen } \\
\text { relatif au } \\
\text { niveau) }\end{array}$ & $\begin{array}{c}\text { H3 : Compétences } \\
\text { (taux de jeunes se } \\
\text { disant utilisés à leur } \\
\text { niveau de compétences) }\end{array}$ & $\begin{array}{c}\text { H4 : Satisfaction } \\
\text { (taux de jeunes se disant } \\
\text { satisfaits de leur situation } \\
\text { prof. à la date d'enquête) }\end{array}$ & $\begin{array}{c}\text { H4 : Recherche } \\
\text { d'emploi } \\
\text { (taux de jeunes } \\
\text { disant chercher } \\
\text { un autre emploi) }\end{array}$ \\
\hline $\begin{array}{l}\text { Indice de concentration } \\
\text { de la formation (Gf) }\end{array}$ & 0.12 & 0.63 & 0.21 & -0.18 \\
\hline $\begin{array}{l}\text { Taux d'emplois de bon } \\
\text { niveau à l'issue } \\
\text { de la formation }\end{array}$ & 0.43 & 0.71 & 0.51 & -0.37 \\
\hline $\begin{array}{l}\text { Taux de CDI (contrat } \\
\text { à durée indéterminée) } \\
\text { à l'issue de la formation }\end{array}$ & 0.44 & 0.59 & 0.46 & -0.36 \\
\hline $\begin{array}{l}\text { Nombre de mois } \\
\text { de chômage }\end{array}$ & -0.50 & -0.50 & -0.60 & 0.49 \\
\hline
\end{tabular}

Source : Enquête Génération 98 du Céreq, formations de niveau supérieur, effectifs de sortants non pondérés $>30$.

Note de lecture : la corrélation entre l'indice de concentration des formations et le niveau de salaire moyen obtenu par les jeunes et rapporté à celui des formations de même niveau est, pour l'ensemble des formations supérieures, de 0.12 , ce qui est faible ; celle entre l'indice de concentration et le taux de jeunes se disant utilisés à leur niveau de compétence est de 0.63 , ce qui est beaucoup plus élevé. 
trois questions binaires : «Êtes vous utilisé à votre niveau de compétences? » (H3, col. 2), «Votre situation vous convient-elle? » et «Recherchez-vous un autre emploi ?» (H4, col. 3 et 4$)$.

Les variables supposées influencer les résultats sont les mêmes pour les quatre modèles :

- Le niveau et la spécialité de la formation du jeune ainsi que son sexe et son origine sociale ;

- L'indice de concentration de sa formation (proxy de l'avantage compétitif attribué à cette formation) ;

- La correspondance entre le niveau de sa formation et celui de son emploi ;

- Les caractéristiques de sa situation de travail à la date de l'enquête, et notamment le nombre de mois travaillés qui est à la fois un indicateur de chômage et d'expérience accumulée. Sont également pris en compte l'ancienneté dans son emploi actuel, son type de contrat de travail et le quota d'heures travaillées ;

- Dans le cas des modèles logit, le logarythme de son salaire pour contrôler les effets de la rémunération sur les variables de perception.

Les résultats (tableau 3) montrent que l'indice de concentration exerce une influence sur les quatre indicateurs testés. Son coefficient est toujours significatif et dans le sens prévu par nos hypothèses.

Concentration et salaire (H2) : Le salaire croît avec le niveau d'études, l'ancienneté et l'expérience professionnelle et il est plus élevé lorsque les conditions d'emploi sont stables. À niveau d'emploi donné, avoir un emploi déclassé est pénalisant alors qu'au contraire obtenir un emploi de niveau supérieur (premières promotions) s'accompagne d'une augmentation de salaire. Enfin, les femmes obtiennent des salaires inférieurs à ceux des hommes $(-10 \%)$ et les jeunes issus de milieux favorisés ont un avantage salarial $(+3 \%$, $+2 \%$ ), toutes choses égales par ailleurs.

Les jeunes qui sortent d'une formation aux débouchés concentrés ont, toutes choses égales par ailleurs, et notamment à situation comparable vis-à-vis de la stabilité, du déclassement et de l'ancienneté, des salaires en moyenne supérieurs à ceux qui, au contraire, sortent de formations aux débouchés dispersés (écart maximum de $+26 \%$ ). Ce résultat valide l'hypothèse $\mathrm{H} 2$ et confirme les résultats de nos précédents travaux (Béduwé, Espinasse, Vincens, 2007). Les jeunes qui ont pu faire valoir leur avantage compétitif ont accédé aux emplois les mieux rémunérés. On a vu avec l'exemple des Docteurs que ces emplois peuvent être ceux directement prévus par la formation (chercheurs) mais pas uniquement (ingénieurs).

Concentration et utilisation des compétences (H3) : L'appréciation des jeunes quant à l'utilisation de leurs compétences est subjective. Elle peut dépendre d'autres facteurs que l'emploi occupé. Ainsi, avoir un emploi déclassé, un contrat d'intérimaire ou une ancienneté très longue diminuent la probabilité de se sentir utilisé à son niveau de compétence, tandis qu'un salaire plus important, l'expérience accumulée et le statut d'indépendant l'augmentent. Cela étant, plus la formation des jeunes est concentrée et plus ils s'estiment utilisés à leur niveau de compétence. H3 est validée. L'avantage compétitif attribué à certaines formations pour certains emplois repose bien sur la nature des compétences acquises. La formation joue donc un rôle explicite dans l'accès à ces emplois particuliers et les jeunes y jugent leurs compétences mieux utilisées.

Concentration et satisfaction (H4) : Être déclassé diminue la satisfaction vis-à-vis de la situation professionnelle $(-3 \%)$ et augmente la probabilité de chercher un autre emploi $(+2 \%)$. Mais ce sont surtout les conditions de travail (stabilité du contrat, temps partiel ou salaire) qui sont déterminantes. On note que la satisfaction diminue lorsque l'ancienneté dans l'emploi s'allonge. À conditions de travail égales, plus leur niveau de diplôme est élevé, plus les jeunes se sentent insatisfaits et décidés à changer d'emploi. Ceci est sans doute lié à l'ambition et les attentes créées par l'obtention d'un niveau de diplôme élevé. Enfin, les filles, dont on a vu qu'elles étaient moins bien payées et moins souvent utilisées à leur niveau de compétence que les garçons sont, sans surprise, moins satisfaites et plus à la recherche d'un autre emploi. L'origine sociale est sans effet sur ces critères subjectifs.

Cela étant, les jeunes issus de formations concentrées sont plutôt plus satisfaits de leur situation et cherchent moins à en changer, toutes choses égales par ailleurs, que les jeunes issus de formations aux débouchés dispersés. Ils avaient un avantage compétitif qu'ils ont pu valoriser en accédant, collectivement, aux emplois 
Tableau 3

Influence de la concentration sur les indicateurs de résultat

\begin{tabular}{|c|c|c|c|c|}
\hline & $\begin{array}{c}\text { H2 : Régression } \\
\text { log (salaire } \\
\text { mensuel) } \\
\text { (1) }\end{array}$ & $\begin{array}{c}\text { H3 : Analyse } \\
\text { logistique } \\
\text { Utilisé à son niveau } \\
\text { de compétences }\end{array}$ & $\begin{array}{c}\mathrm{H} 4 \text { : Analyse } \\
\text { logistique } \\
\text { Satisfaction quant à } \\
\text { la situation actuelle }\end{array}$ & $\begin{array}{l}\text { H4 : Analyse } \\
\text { logistique } \\
\text { Recherche d'un } \\
\text { autre emploi }\end{array}$ \\
\hline Constant & 6.77 & $+0.68(2)$ & $0.73(2)$ & $+0.24(2)$ \\
\hline Genre (ref : femme) & +0.10 & $-0.05(3)$ & $-0.02(3)$ & $+0.02(3)$ \\
\hline $\begin{array}{l}\text { Père Cadre ou Indép. (ref : autre CSP) } \\
\text { Mère Cadre ou Indép. (ref : autre CSP) }\end{array}$ & $\begin{array}{l}+0.03 \\
+0.02 \\
\end{array}$ & $\begin{array}{c}\text { Ns } \\
\text { ns }\end{array}$ & $\begin{array}{l}\text { Ns } \\
\text { ns }\end{array}$ & $\begin{array}{l}\text { Ns } \\
\text { ns }\end{array}$ \\
\hline \multicolumn{5}{|l|}{ Niveau du diplôme de sortie (ref : Bac +1 ) } \\
\hline Niveau Bac +5 et plus & +0.38 & -0.06 & -0.11 & +0.11 \\
\hline Niveau Bac $+3,4$ & +0.21 & -0.03 & -0.05 & +0.04 \\
\hline Niveau Bac +2 & Ns & ns & -0.02 & +0.03 \\
\hline \multicolumn{5}{|c|}{ Spécialité de formation (ref : sciences \& industrie) } \\
\hline Lettres, Sciences Hum, Soc \& Droit & -0.06 & -0.03 & -0.04 & +0.03 \\
\hline Gestion \& Communication & +0.03 & Ns & -0.02 & +0.04 \\
\hline Santé & +0.12 & +0.06 & -0.04 & -0.06 \\
\hline Services & -0.07 & +0.03 & ns & ns \\
\hline $\begin{array}{l}\text { Expérience (en mois) avant emploi } \\
\text { actuel }\end{array}$ & +0.005 & $+0.002(4)$ & ns & Ns \\
\hline Ancienneté dans l'emploi en 2001 & +0.005 & -0.002 & -0.002 & +0.003 \\
\hline Salaire mensuel & & +0.001 & +0.001 & -0.0007 \\
\hline \multicolumn{5}{|l|}{ Horaires de travail (ref : temps plein) } \\
\hline Temps partiel & -0.44 & ns & -0.07 & +0.09 \\
\hline Non déclarés (indépendants) & +0.33 & +0.14 & +0.11 & -0.12 \\
\hline \multicolumn{5}{|l|}{ Contrat de travail (ref : CDI ou fonctionnaire) } \\
\hline CDD & -0.14 & ns & -0.17 & +0.16 \\
\hline Intérim & -0.05 & -0.17 & -0.29 & +0.31 \\
\hline Mesure pour l'emploi & -0.32 & -0.04 & -0.16 & +0.11 \\
\hline \multicolumn{5}{|c|}{ Correspondance entre niveau d'emploi et niveau de formation (ref : bien classés) } \\
\hline Déclassé & -0.16 & -0.12 & -0.03 & +0.02 \\
\hline Surclassé & +0.19 & ns & ns & ns \\
\hline Indice de concentration & +0.26 & $+0.32(5)$ & $+0.20(5)$ & $-0.24(5)$ \\
\hline $\mathrm{N}$ & 26586 & 26586 & 26586 & 26586 \\
\hline R2 / log(pseudovraissemblance) & 0.57 & -15914 & -14845 & -14270 \\
\hline
\end{tabular}

Source : Enquête Génération 98 du Céreq, sortants de formations de niveau strictement supérieur au Bac, en emploi en avril 2001.

(1) : Il s'agit d'une fonction de gains où le salaire expliqué est le salaire moyen mensuel obtenu en avril 2001. Lire : Toutes choses égales par ailleurs (c'est-à-dire compte tenu de toutes les variables introduites dans la régression), les hommes gagnent $10 \%$ de plus que les femmes, les étudiants dont le père était Cadre ou Indépendant $3 \%$ de plus, etc. et les étudiants sortis d'une formation où l'indice de concentration est maximum (0.99) gagnent en moyenne $26 \%$ de plus que ceux sortis d'une formation dont l'indice est minimum (0.76).

(2) Lire : La probabilité de référence de se dire utilisé à son niveau de compétence est de 0.68 , celle d'être satisfait de sa situation actuelle est de 0.73 et de chercher un autre emploi est de 0.24 ; ces probabilités sont calculées pour toutes les situations de référence (femme, Bac +1 , Père et Mère non Cadre ou Indépendant, etc.) et les valeurs moyennes de l'expérience acquise avant l'emploi en avril 2001 (7,7 mois), de l'ancienneté dans l'emploi en avril 2001 (20 mois), du salaire en avril 2001 (1392 euros) et d'un indice de concentration (0.88).

(3) Lire : Être un homme diminue la probabilité de resp. $5 \%, 2 \%$ et $2 \%$ de resp. se dire utilisé à son niveau de compétences, satisfait de sa situation actuelle et à la recherche d'un autre emploi, toutes choses égales par ailleurs.

(4) Lire : un mois d'expérience professionnelle en plus (en dehors de l'ancienneté dans l'emploi) augmente la probabilité de se dire utilisé à son niveau de compétences de $0.2 \%$, toutes choses égales par ailleurs.

(5) Lire : sortir d'une formation dont l'indice de Gini est supérieur de 0.1 augmente la probabilité de se dire utilisé à son niveau de compétences. 
qu'ils jugeaient préférables et qui s'avèrent effectivement plus satisfaisants. La dernière hypothèse, qui postule un lien positif entre satisfaction et concentration, se trouve ainsi également validée.

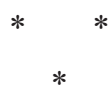

L'indice de concentration des emplois occupés constitue une caractéristique de l'insertion professionnelle des sortants d'une formation. L'analyse du marché du travail que nous proposons montre que la valeur de l'indice est très liée à l'avantage compétitif que possèdent les sortants de cette formation à l'égard de quelques emplois. Plus l'indice est élevé, plus les jeunes ont pu faire valoir leur avantage compétitif et plus il est probable qu'ils aient accédé aux meilleurs emplois parmi ceux qui leur étaient accessibles sur une période donnée. L'observation confirme effectivement que plus la concentration s'élève, plus les emplois occupés sont bien rémunérés, les compétences acquises en formation bien valorisées et les jeunes plus satisfaits de leur situation professionnelle.

L'indice de concentration apparaît donc comme une bonne clé d'entrée dans l'analyse de l'insertion professionnelle.

Il permet de clarifier le jeu des concurrences entre formations. Quant les jeunes issus d'une formation se sont majoritairement tournés vers quelques emplois, on peut considérer que cette formation a permis à ses formés d'accéder aux emplois qu'ils jugeaient, collectivement, préférables. L'indice de concentration fait ressortir ce que l'on peut appeler le paradoxe concurrentiel : plus l'avantage compétitif est élevé et plus sa concrétisation par des recrutements dépend de la demande émanant des emplois pour lesquels existe cet avantage. Au contraire, un avantage plus faible, fondé essentiellement sur des compétences partagées et qui, de ce fait, donne accès à un plus grand nombre d'emplois, réduit le risque lié à une demande insuffisante tout en accroissant le risque lié à la concurrence.

L'indice de concentration permet une mesure du lien qu'entretient une formation avec les emplois. Plus l'indice est élevé et plus la formation a joué un rôle dans l'accès à des emplois satisfaisants. La difficulté majeure des analyses en termes d'adéqua- tion, qui reposent sur un raisonnement binaire, i.e l'emploi est adéquat ou non, se trouve ainsi écartée. L'analyse des divers types de compétences, acquises en formation et requises par l'emploi, suggère que les possibilités d'adaptation sont beaucoup plus grandes, et c'est ce qu'exprime la notion d'avantage compétitif. Les sortants de plusieurs formations peuvent être concurrents pour les mêmes emplois sans que la qualité de l'appariement en pâtisse. L'emploi convient à ces formés et leurs formations conviennent aux employeurs. Au paradigme d'une mise en correspondance normative peut se substituer le paradigme de la convenance $^{13}$ (Vincens, 2008).

L'indice de concentration constitue ainsi un élément intéressant pour l'évaluation multicritères d'une formation. Il permet de relativiser le rôle de la formation et de mieux comprendre comment s'est opérée l'insertion professionnelle des formés. Associé aux indicateurs de résultat d'insertion classiques, il participe d'une évaluation qui peut déboucher sur des recommandations utiles à l'évolution des formations. Une formation dont l'indice de concentration est plus élevé qu'une autre signale qu'elle a, en toute probabilité, joué un rôle important dans l'accès de ses jeunes aux meilleurs emplois auxquels ils pouvaient prétendre, compte tenu des conditions du marché. Pour autant, ce n'est pas un indicateur de résultat susceptible de classer les formations sur une échelle de réussite. D'abord parce qu'évaluer ne signifie pas forcément classer (voir par exemple Perret, 2010, sur la question des indicateurs). Et, ensuite, parce que notre analyse confirme, à l'instar de la plupart des analystes de l'insertion professionnelle (par exemple Giret et alii, 2010 ; Ourliac, 2010), qu'aucun critère considéré isolément ne peut réellement tenir ce rôle.

\footnotetext{
13 J. Vincens (2008, p. 167) : «Chacun recherche ce qui peut lui convenir compte tenu de ses atouts et de ses contraintes; et ce qui convient est ce qui donne à chacun le meilleur rapport avantages/coûts. Fondamentalement, chaque contrat de travail est un couplage unique, résultat d'une double sélection sous contraintes : sélection de l'emploi par l'individu et de l'individu par l'employeur; c'est ce qu'exprime l'idée de convenance. On ne peut limiter cette convenance à une correspondance entre une formation et un intitulé d'emploi, comme le voudrait le paradigme de l'adéquation normative, sans appauvrir l'analyse des raisons qui conduisent au contrat. »
} 


\section{Bibliographie}

Affichard J. (1981), « Quels emplois après l'école : la valeur des titres scolaires depuis $1973 »$, Économie et Statistique, 173, pp. 7-26.

Affichard J., Combes M.-C., Grelet Y. (1992), «Apprentis et élèves des lycées professionnels : où sont les emplois stables ? », Formation Emploi n ${ }^{\circ} 38$, pp. 9-28.

Béduwé C, Vincens J. (2010), «Quels indicateurs pour quelle évaluation des formations? », Actes des XVII journées d'études sur les données longitudinales dans l'analyse des marchés du travail, Marseille, pp. 263-282.

Béduwé C., Espinasse J.-M., Vincens J. (2007), « De la formation professionnelle à la professionnalité d'une formation », Formation Emploi n ${ }^{\circ}$ 99, pp. 103-121.

Béduwé C., Espinasse J.-M. (2009), Pour une approche statistique des relations entre formations et emplois, rapport de recherches pour la dgesco, ministère de l'Éducation nationale, CPC documents $n^{\circ} 2009 / 10,84$ p.

Béduwé C., Giret J.-F. (2010), "Mismatch of vocational graduates: what penalty on french labour market?", Journal of vocational behavior(2010), doi/10.1016:j;jvb;2010.09.003.

Bourdon J., Giret J.-F, Goudard M., (2011), Peut-on classer les universités en fonction de leur performance d'insertion? documents de travail de l'iredu, dt 2011/2.

Bruyère M., Lemistre P. (2010), « La formation professionnelle des jeunes : quelle valorisation de la spécialité ? », Revue d'Économie politique, 2010/3, vol. 120.

Cedefop (2010), The skill matching challenge. Luxembourg. Publications office of the european union.

Chardon O. (2005), «La spécialité de formation joue un rôle secondaire pour accéder à la plupart des métiers 》, Économie et Statistique n ${ }^{\circ}$ 388-389.
Dumartin S. (1997), «Formation emploi: quelle adéquation?», Économie et Statistique, 303, pp. 59-80.

Giret J.-F., Lopez A., Rose J. (2005), Quelles formations pour quels emplois?, ouvrage collectif, Paris, La Découverte, $384 \mathrm{p}$.

Giret J.-F. \& Moullet S. (2008), « Une analyse de la professionnalisation des formations de l'enseignement supérieur à partir de l'insertion de leurs diplômés », net.doc $\mathrm{n}^{\circ} 35$, Céreq.

Heijke H., Matheeuwsen A. \& Willems E. (2003), "Clustering educational categories in a heterogeneous labour market", Education economics, 11(1), pp. 89-108.

Mc Guinness (2006), "Overeducation in the labour market", Journal of economic surveys, vol. 20, $\mathrm{n}^{\circ} 3$.

Ourliac G. (2010), «Évaluer une politique publique à travers ses résultats ou ses enjeux ? L'exemple des politiques régionales de formation professionnelle » in L'évaluation des politiques publiques, le développement d'une nouvelle culture, ouvrage coordonné par P. Braconnier et G Cauquil, CNDP, pp 153-159.

Perret B. (2010), «Évaluer une politique publique à travers ses résultats ou ses enjeux ? L'exemple des politiques régionales de formation professionnelle » in L'évaluation des politiques publiques, le développement d'une nouvelle culture, ouvrage coordonné par P. Braconnier et G. Cauquil, CNDP, pp. 135-141.

Plassard J.-M., Thii Tanh Nhu Tran (2009), «L'analyse de la suréducation: l'escroquerie scolaire enfin démasquée ou beaucoup de bruit pour rien ? », Revue d'économie politique, vol. 119, $\mathrm{n}^{\circ} 5$ sept-octobre.

Sattinger M. (1993), "Assignement models of the distribution of earnings", Journal of Economic Litterature. vol. xxxi, june, pp. 831-880. 
Stankiewicz F. (sous la direction de) (1998), Travail, compétences et adaptabilité, L'Harmattan.

Stasz C. (2001), “Assessing skills for work: two perspectives", Oxford economic papers, $\mathrm{n}^{\circ} 3$.
Thurow L. C. (1975), Generating inequality, Basic books inc, publishers New York.

Vincens J. (2008), «Évolution de l'enseignement supérieur: persistance des paradoxes », Cahier $d u$ Lirhe, $\mathrm{n}^{\circ} 14,260 \mathrm{p}$.

\section{Résumé}

\section{L'indice de concentration : \\ une clé pour analyser l'insertion professionnelle et évaluer les formations}

Catherine Béduwé et Jean Vincens

L'évaluation externe des formations repose sur les résultats de l'insertion professionnelle des sortants, appréciée à l'aide de différents critères. La répartition des débouchés d'une formation sur les différents emplois occupés est ici appréhendée au moyen d'un indice de concentration (dit " gini »). Ce critère rend compte de la position concurrentielle d'une formation sur le marché du travail, position dépendante de l'avantage compétitif qu'elle possède à l'égard de quelques emplois. On peut donc avancer que les formations dont les débouchés sont concentrés obtiendront en moyenne de meilleurs résultats d'insertion, notamment en termes de salaire, d'utilisation des compétences et de satisfaction. Nos résultats montrent que ces hypothèses sont validées par les données de l'enquête Génération 98 du Céreq. L'indice de concentration peut ainsi contribuer à expliquer les différences d'insertion entre formations et identifier les moyens susceptibles d'améliorer leurs résultats.

\section{Mots clés}

Insertion professionnelle, rendement de l'éducation, statistique de l'éducation

Journal of Economic Literature: J 24, C 16 